\title{
Analysis on the Error Types and the Causes of the Construction of “X 什么(Y)+都+Z"
}

\section{Siyun Li*}

Kegan Dialect and Language Application Research Center, Nanchang University, Nanchang 330000, Jianggxi Province, China

*Corresponding author: Siyuan Li, $916911790 @ q q . c o m$

\begin{abstract}
Using intermediate and advanced Chinese students as the research subjects, HSK corpus and questionnaire surveys were used to explore the error types in the construction of "X 什么 $(\mathrm{Y})+$ 都+Z." The types of errors can be divided into component errors, relationship errors between components, and collocation errors between components and sentences. The main reasons for the errors are the particularity of the construction, the immature development of construction teaching, the negative transfer of students' mother tongue, as well as the generalization and avoidance of the target language.
\end{abstract}

Keywords: $\mathrm{X}$ 什么 $(\mathrm{Y})+$ 都+Z; Error type; Teaching Chinese as a foreign language

Publication date: August 2021; Online publication: August 30, 2021

\section{Collection and sorting of errors}

Error analysis is a systematic analysis of errors produced by learners in the process of second language acquisition via studying their sources, revealing the interlanguage system of learners, and thus understanding the process and rules in second language acquisition ${ }^{[1]}$. In this article, the sentences written by students that were learning the construction of " $\mathrm{X}$ 什么 $(\mathrm{Y})+$ 都 $+\mathrm{Z}$ " were retrieved from the HSK dynamic composition corpus of Beijing Language and Culture University, and questionnaires were compiled according to their error types to further explore the types and causes of their errors in learning the construction. This article had only analyzed syntactic errors. Semantic and pragmatic errors, pronunciation, Chinese characters, punctuation errors, and other errors would be further discussed in the future.

\subsection{Interlanguage corpus errors}

\subsubsection{Corpus collected}

After manual screening, 55 incorrect sentences containing the construction of “ $\mathrm{X}$ 什么 $(\mathrm{Y})+$ 都+Z” were retrieved from the HSK corpus.

\subsubsection{Corpus statistics}

Among the 55 sentences, one of the sentences had multiple errors, thus a total of 69 errors were accounted for. Among them, the largest errors were of "都" and " $Z$ "; following that, the errors of " $X$ " and " $Y$ "; the internal and external errors of components were the least. 


\subsection{Errors in questionnaires}

\subsubsection{Research purpose}

The statistical corpus was taken as the basis of the questionnaire. Combined with relevant points from textbooks for international students, the questionnaire was designed. The test results were statistically analyzed to determine the understanding and application of the construction among international students.

\subsubsection{Background of the respondents and questionnaire}

The respondents were intermediate and advanced Chinese students, most of whom had learned this structure or similar structures in textbooks. A total of 70 questionnaires were distributed via Questionnaire Star with 60 questionnaires being recovered; the recovery rate was $86 \%$ while the answer rate was $100 \%$.

\subsubsection{Setting of questions for the questionnaire}

In order to ensure the reliability and validity of the questionnaire, the questionnaire was prepared based on the error sentences in the HSK corpus. The questions were all from the error corpus of BCC corpus, CCL corpus, and HSK corpus with a total of 30 questions within 15 minutes.

\subsubsection{Analysis of the survey results}

According to the survey results, the errors in the corpus have been confirmed by the questionnaires. First, except for a small error rate of "Y" and “什么," there were language points with error rate higher than 50\% in other errors. Second, the error rates of auxiliary verbs in " $X$ " and NPs (noun phrases) were the highest; both above $70 \%$. Third, the error rate of "都" was more than $50 \%$. Fourth, the error rates of "Z" and NPs were also more than $50 \%$. It can be seen that the aforementioned errors were easily made by students in the corpus and questionnaire.

\section{Analysis of the types of errors}

\subsection{Component errors}

\subsubsection{Error of " $X$ "}

(1) Misadding components in " $X$ "

Example 1. *她才思敏捷，想说什么都有理有据。(Questionnaire)

Note: Sentences which are marked with an asterisk (*) are error sentences, and the source of each of these sentences is indicated in brackets.

In Example 1, “说什么都有理有据” shows the characteristics of her quick thinking. The verb “想,” which means “hope and plan,” should be deleted as it is not necessary.

(2) Omission of "X"

Example 2. * 我自己觉得我是失败的人。所以什么事情都没有兴趣。(HSK corpus)

" $\mathrm{X}$ " is often the action or state of a NP; hence, " $\mathrm{X}$ " is often a V (verb) or VP (verb phrase). Students sometimes omit the NP of the former clause in the latter clause, thus leaving out the V or VP of the action in NP. The NP in Example 2 is the first person “我.” In the latter sentence, the action issuer 
“我” and “不感兴趣的事” are agent relations and should be replaced with “我对什么事情都没有 兴趣.”

\subsubsection{Error of "Y"}

The relationship between $\mathrm{X}$ and $\mathrm{Y}$ is usually a verb-object relationship, and at times, students add redundant components after the object. This article deems it as an incorrect addition of components in "Y."

Example 3. * 那时候的我无比难受, 用什么话来都表达不出我的心理状态。(HSK corpus)

As seen in Example 3, “来” in “用什么话来” is a verb, indicating that the former is a method, direction, or attitude, and the latter is a purpose. This sentence is incorrect; “来” should be deleted.

\subsubsection{Error of “都”}

(1) “都” is wrongly replaced by other components

Example 4. *这家餐馆的菜太难吃了, 我们说什么也要换一家。(Questionnaire) Example 5. *子女有新的观念，做什么就自有主张，不太问父母。(HSK corpus)

The usage of “都” and “也” are similar. It can be used interchangeably when the two things are the same, but “也” is more euphemistic than “都.” In Example 4, this sentence explains that “太难 吃." The second half of the sentence expresses a strong desire to turn things around. The use of “都” is more in line with pragmatic needs. The adverb “就” in Example 5 means that it will happen in a short time, which is not in line with the meaning of the sentence. It should be changed to “都," which provides a light transition.

(2) Omission of “都”

Example 6. *我认为，他对工作，家庭的热情告诉我做什么事情不应该忘记 “认真”。(HSK Corpus)

Omission of “都” is also a common error. In Example 6, “都” is not used to connect the previous sentences with the subsequent ones; thus, the sentence is unable to express a light transition. "都” should be added; that is, "you should not forget "认真" when doing anything."

\subsubsection{Error of " $Z$ "}

(1) Incorrect composition in " $Z$ "

Example 7. *The neighbor's children are so naughty that their parents won't be satisfied with anything they want to do. (Questionnaire)

Example 7 have errors of adverbs in “Z." The auxiliary verb “不会” indicates that the negative form in the future should be changed to “不能.”

(2) Omission of components in "Z"

Example 8. *我们知道放弃什么, 选择什么都给我们带来负面的影响。(HSK corpus) 
In Example 8, “Z” lacks an adverbial, thus it is better to add the auxiliary verb “会," which indicates possibility.

(3) Misaddition of components in " $Z$ "

Example 9. *在这里很多中国朋友帮助我。比如说去买东西啊, 去办什么事儿他们都要帮我。 (HSK corpus)

In Example 9, the adverbial in “Z” has been wrongly added. “去办什么事儿他们都帮我” describes the friendly attitude of the friends toward “我." There is no need to use the auxiliary verb “要” which means “必须, 应该”; it is redundant and should be deleted.

\subsection{Relationship errors between components}

\subsubsection{Mismatch of " $X$ " and " $Y$ "}

Example 10. *战士们遇什么险恶的情况都信心十足, 毫不动摇。(Questionnaire)

Example 11. *我觉得吸烟对什么方向都没好处。(Questionnaire)

In Example 10, “遇” in “遇什么险恶的情况” should be replaced with the verb phrase complement “遇到.” In Example 11, “对什么方向” is inconsistent with the meaning of the sentence, and should be changed to “对什么方面."

\subsubsection{Wrong order of " $X$ " and " $Y$ " and “什么"}

Example 12. *小女孩什么东西都看到充满怜爱。(Questionnaire)

Example 13. *看母亲的这样的情况后, 我朋友像她一样养成了做事情什么都凑合着做的坏习惯。 (HSK corpus)

Example 12 shows that the student had misplaced "X" and “什么”; that is, the wrong order of “看到” and “什么.” It should be changed to “看到什么东西都充满怜爱.” In Example 13, the wrong order of “什 么” and “事情” should be changed to “做什么事情都凑合着做的坏习惯.”

\subsection{Collocation errors between components and sentences}

\subsubsection{Errors of NP in the sentence where the construction is located}

Example 14. * 只一起过了几年的朋友们的爱好, 需要什么东西都知道, 却不知道过了一生的父 母到底需要什么东西。(HSK corpus)

The error in Example 14 is the omission of NP. In “需要什么东西都知道,” “我” should be added before “知道."

\subsubsection{Misuse of the sentence “是.......的”}

Example 15. * *法律是对什么人都有公平的道理。(HSK corpus) 
“是.......的” is often used in the construction of sentences. Students often make mistakes in terms of missing components. For example, the misuse of “是.......的” in “是对什么人都有公平的道理” should be changed to “是对什么人都有公平的道理的.”

\section{Analysis of the cause of errors}

\subsection{Particularity of the construction of " $X$ 什么(Y)+都 $+Z$ "}

The meaning and usage of this construction are complex. First, there exists a verb-object relationship between "Y" and "X," in which the language units that can replace "X" and "Y" have their own characteristics. The second is the usage of “都.” Adverbs such as “都,” “也,” and “就” are related but different. Thirdly, the usage of " $Z$ " is complicated, in which the usage of adverbial and predicate in place of " $Z$ " are diversified. These are the mistakes and difficulties faced among international students.

On the other hand, the construction is related to but different from similar structures, such as “无论/不 论/不管......都......” Compared with “X 什么(Y)+都+Z,” the latter has no conjunction “无论/不管,” thus it is not as strong as the former. Comparing “连......都......" with " $\mathrm{X}$ 什么(Y)+都+Z," the latter has no conjunction “连," so the contrast is not as strong as the former. Due to poor understanding of contexts, students are prone be confused in its usage.

\subsection{Teaching aspects}

In terms of teaching methods, teachers generally use the term "fixed structure" to explain and guide students to understand the meaning of the whole construction; from the generic usage of “什么” to the light transition of “都." Students do not systematically learn the key points, difficult points, and easy mixing points in the construction. In terms of textbook compilations, the selection and layout of grammar in TCSL are excellent, which have been the key to its success ${ }^{[2]}$. However, the current textbooks lack systematic and scientific explanation as well as the practice of construction, thus making it difficult for students to think in chunks in order to understand the whole construction.

\subsection{Learning aspects}

\subsubsection{Negative transfer of native language}

The negative transfer of native language refers to the reliance on the knowledge of the learners' native language when they are not familiar with the rules of the target language ${ }^{[1]}$. Students who use this construction are generally of intermediate and advanced Chinese level, in which they have a certain foundation, thus the negative transfer of their mother tongue is less.

\section{Example 16. *我们要做什么事都要经过思虑。(HSK corpus)}

Example 16 shows the semantic error of verbs by foreign students whose mother tongue is Thai. “ พิจารณา” in Thai also means “思虑” and “思考.” In Mandarin, “思虑” means deep thinking whereas “思 考” means neutral thinking activities. Example 16 wants to express “做事情都要经过思考,” thus “思虑” should be replaced with “思考.”

\subsubsection{Negative transfer of target language knowledge}

The negative transfer of target language knowledge refers to the error caused by the learners' incorrect application of their limited target language knowledge; that is, the new language phenomenon of the target language by analogy ${ }^{[1]}$. 
Example 17. *我觉得学习语言时比什么最重要的就是口语。(HSK corpus)

Example 17 shows that the adverb “最” that means “极端, 胜过其余” have been misused as the absolute meaning of “ $\mathrm{X}$ 什么(Y)+都+Z” has not been fully mastered. “最” should be replaced with “都.”

\subsubsection{Avoidance}

The variable and constant terms of the construction mandate many rules; hence, students would tend to use other expressions to avoid using this construction. This psychological avoidance results in reduced practice and error correction in the process of acquisition, which bound to increase the probability of errors.

\section{Conclusion}

This article analyzed the errors of foreign students in their acquisition of the construction of " $\mathrm{X}$ 什么(Y)+ 都+Z." Based on the statistics of errors from HSK corpus and the questionnaires, the types of errors can be divided into three categories: component errors, relationship errors between components, and collocation errors between components and sentences. The main reasons for these errors are the particularity of the construction, the immature development of the construction teaching, the negative transfer of students' mother tongue, as well as the generalization and avoidance of the target language.

\section{Disclosure statement}

The author declares that there is no conflict of interest.

\section{References}

[1] Liu K, 2000, Introduction to Education as a Foreign Language. Beijing Language and Culture University Press, Beijing, 191, 195

[2] LV W, 2002, Principles and Strategies for Ranking Grammar Items in Teaching Chinese as a Foreign Language. Chinese Teaching in the World, (4): 4, 86-95. 